\title{
Impact of enzymatic digestion on bacterial community composition in CF airway samples
}

Kayla M. Williamson ${ }^{1}$, Brandie D. Wagner ${ }^{\text {Corresp., }}{ }^{1,2}$, Charles E. Robertson ${ }^{3}$, Emily J. Johnson ${ }^{2,4}$, Edith T. Zemanick $^{2}$, J. Kirk Harris ${ }^{2}$

1 Department of Biostatistics and Informatics, Colorado School of Public Health, University of Colorado at Denver, Aurora, Colorado, United States

2 Department of Pediatrics, School of Medicine, University of Colorado at Denver, Aurora, Colorado, United States

3 Division of Infectious Diseases, School of Medicine, University of Colorado at Denver, Aurora, Colorado, United States

4 Multicare Tacoma Family Medicine, Tacoma, Washington, United States

Corresponding Author: Brandie D. Wagner

Email address: brandie.wagner@ucdenver.edu

Background. Previous studies have demonstrated the importance of DNA extraction methods for molecular detection of Staphylococcus, an important bacterial group in cystic fibrosis (CF). We sought to evaluate the effect of enzymatic digestion (EnzD) prior to DNA extraction on bacterial communities identified in sputum and oropharyngeal swab (OP) samples from patients with CF. Methods. DNA from 81 samples (39 sputum and 42 OP) collected from 63 patients with CF was extracted in duplicate with and without EnzD. Bacterial communities were determined by rRNA gene sequencing, and measures of alpha and beta diversity were calculated. Principal Coordinate Analysis (PCoA) was used to assess differences at the community level and Wilcoxon Signed Rank tests were used to compare relative abundance (RA) of individual genera for paired samples with and without EnzD. Results. Shannon Diversity Index (alpha-diversity) decreased in sputum and OP samples with the use of EnzD. Larger shifts in community composition were observed for OP samples (beta-diversity, measured by Morisita-Horn), whereas less change in communities was observed for sputum samples. The use of EnzD with OP swabs resulted in significant increase in RA for thegenera Gemella $(p<0.01)$, Streptococcus $(p<0.01)$, and Rothia $(p<0.01)$. Staphylococcus $(p<0.01)$ was the only genus with a significant increase in RA from sputum, whereas the following genera decreased in RA with EnzD: Veillonella $(p<0.01)$, Granulicatella $(p<0.01)$, Prevotella $(p<0.01)$, and Gemella $(p=0.02)$. In OP samples, higher RA of Gram-positive taxa was associated with larger changes in microbial community composition. Discussion. We show that the application of EnzD to CF airway samples, particularly OP swabs, results in differences in microbial communities detected by sequencing. Use of EnzD can result in large changes in bacterial community composition, and is particularly useful for detection of Staphylococcus in CF OP samples. The enhanced identification of Staphylococcus aureus is a strong indication 
to utilize EnzD in studies that use OP swabs to monitor CF airway communities. 
1 Impact of enzymatic digestion on bacterial community composition in CF airway samples

2 Kayla M. Williamson ${ }^{1}$, Brandie D. Wagner ${ }^{1,2}$, Charles E. Robertson ${ }^{3}$, Emily J. Johnson²*, Edith 3 T. Zemanick ${ }^{2}$, and J. Kirk Harris ${ }^{2}$

4

$5{ }^{1}$ Department of Biostatistics and Informatics, Colorado School of Public Health, University of

6 Colorado School of Medicine, Aurora, Colorado, United States of America

7 2Department of Pediatrics, University of Colorado School of Medicine, Aurora, Colorado,

8 United States of America

$9{ }^{3}$ Division of Infectious Diseases, University of Colorado, School of Medicine, Aurora, Colorado,

10 United States of America

*Current Address: Multicare Tacoma Family Medicine, Tacoma, Washington, United States of

12 America

Corresponding author:

Brandie Wagner 


\section{Abstract}

Background. Previous studies have demonstrated the importance of DNA extraction methods

for molecular detection of Staphylococcus, an important bacterial group in cystic fibrosis (CF).

We sought to evaluate the effect of enzymatic digestion (EnzD) prior to DNA extraction on bacterial communities identified in sputum and oropharyngeal swab (OP) samples from patients with $\mathrm{CF}$.

Methods. DNA from 81 samples (39 sputum and 42 OP) collected from 63 patients with CF was extracted in duplicate with and without EnzD. Bacterial communities were determined by rRNA gene sequencing, and measures of alpha and beta diversity were calculated. Principal Coordinate Analysis (PCoA) was used to assess differences at the community level and Wilcoxon Signed

Rank tests were used to compare relative abundance (RA) of individual genera for paired samples with and without EnzD.

Results. Shannon Diversity Index (alpha-diversity) decreased in sputum and OP samples with the use of EnzD. Larger shifts in community composition were observed for OP samples (betadiversity, measured by Morisita-Horn), whereas less change in communities was observed for sputum samples. The use of EnzD with OP swabs resulted in significant increase in RA for the genera Gemella $(\mathrm{p}<0.01)$, Streptococcus $(\mathrm{p}<0.01)$, and Rothia $(\mathrm{p}<0.01)$. Staphylococcus $(p<0.01)$ was the only genus with a significant increase in RA from sputum, whereas the following genera decreased in RA with EnzD: Veillonella $(p<0.01)$, Granulicatella $(p<0.01)$, Prevotella $(p<0.01)$, and Gemella $(p=0.02)$. In OP samples, higher RA of Gram-positive taxa was associated with larger changes in microbial community composition.

Discussion. We show that the application of EnzD to CF airway samples, particularly OP swabs, results in differences in microbial communities detected by sequencing. Use of EnzD can result 
42 in large changes in bacterial community composition, and is particularly useful for detection of

43 Staphylococcus in CF OP samples. The enhanced identification of Staphylococcus aureus is a

44 strong indication to utilize EnzD in studies that use OP swabs to monitor CF airway

45 communities. 


\section{Introduction}

Cystic Fibrosis (CF) is an autosomal recessive disease, characterized by chronic airway infection, whose predominant pathogens include Staphylococcus aureus, Pseudomonas aeruginosa and other Gram-negative bacteria (Heijerman, 2005; LiPuma, 2010). OP swabs are commonly used when children are unable to expectorate (Zemanick et al., 2015). This is particularly true for pediatric, non-expectorating subjects with $\mathrm{CF}$ in whom oropharyngeal (OP) cultures are used as a surrogate for lower airway bacteria. (Zemanick et al., 2015). In the pediatric studies, OP swabs have ranged from $30 \%$ to $68 \%$ of samples collected (Armstrong et al., 1996; Zemanick et al., 2010; Wolter et al., 2013; Hoppe et al., 2015) and therefore represent an important sample type in early CF.

Sequencing is becoming more widely used to evaluate bacterial communities in $\mathrm{CF}$ airway samples (Cummings et al., 2016). DNA preparation prior to sequencing, including cell lysis, can have a profound effect on microbial community composition especially in detecting certain organisms such as $S$. aureus(Zhao et al., 2012; Yuan et al., 2012b; Willner et al., 2012; Lozupone et al., 2013; Pérez-losada, Crandall \& Freishtat, 2016). S. aureus in particular has a rigid cell wall that can be hard to rupture, hindering the ability to efficiently extract DNA for molecular detection (Zhao et al., 2012).

Previous studies have indicated that use of enzymatic digestion (EnzD) may enhance the ability to detect Staphylococcus (Schindler \& Schuhardt, 1964; Browder et al., 1965; Yuan et al., 2012a; Zhao et al., 2012; Johnson et al., 2016). Specifically, Yuan et al (2012) performed a comprehensive experiment evaluating multiple DNA extraction methods utilizing human associated bacterial species as well as a mock community. Zhao et al. (2012) found that EnzD increased the yield of Staphylococcus in sputum samples. Similarly, Johnson et al (2016) 
69 determined EnzD improved the sensitivity of sequencing in OP swabs while no differences were

70 observed in sputum samples. Increased sensitivity with EnzD was robust when compared to

71 clinical culture results and qPCR. Furthermore, the work showed that the majority of

72 Staphylococcus was $S$. aureus. It remains unclear, however, how the use of EnzD effects the

remaining bacterial community in clinical CF samples. In this work, we build on these two

previous CF studies to evaluate the effect of DNA extraction using enzymatic digestion (EnzD)

on bacterial communities detected from oropharyngeal swab (OP) and sputum samples collected from patients with $\mathrm{CF}$.

\section{Materials \& Methods}

Patient demographics and samples. Sputum and OP samples were obtained from patients

with CF as part of standard of care for monitoring bacterial infection during routine patient visits.

Further explanation of selection of samples to be used for this study are included in a previous publication(Johnson et al., 2016). Excess specimen was stored frozen at $-80{ }^{\circ} \mathrm{C}$ for molecular assessment of infection. Standard sputum processing protocol was performed for sample homogenization utilizing sputalysin and standard CF culture was performed following CF Foundation guidelines (Burns et al., 1998). The Colorado Multiple Institutional Review Board approved the study (COMIRB 07-0835). Written informed consent was obtained from patients or guardians. Written informed assent was obtained for children 10-17 years of age. extraction platform using the Tissue kit and bacterial card per manufacturer's instructions. EnzD was performed on one replicate of each sample by mixing with lysostaphin (final concentration 
$910.18 \mathrm{mg} / \mathrm{mL})$ and lysozyme $(3.6 \mathrm{mg} / \mathrm{mL})$ and incubated at $37^{\circ} \mathrm{C}$ for 30 minutes. Samples were

92 then digested with proteinase $\mathrm{K}(1.4 \mathrm{mg} / \mathrm{mL})$ and incubated at $65^{\circ} \mathrm{C}$ for ten minutes, then

93 incubated at $95^{\circ} \mathrm{C}$ for 10 minutes. (Zhao et al., 2012; Johnson et al., 2016). Lysozyme and

94 lysostaphin target degradation of the bacterial cell wall by targeting peptidoglycan (lysozyme)

95 and pentaglycine bridges (lysostaphin) (Salazar \& Asenjo, 2007). Lysostaphin is specific to the

96 subset of staphylococci that contain pentaglycine bridges including the human pathogen S.

97 aureus (Trayer \& Buckley, 1970). Proteinase K is a broad specificity endopeptidase that is used

98 to digest proteins (Gradisar et al., 2005).

16S rRNA gene Amplicon Library Construction. Bacterial profiles were determined by

broad-range amplification and sequence analysis of 16S rRNA genes following our previously

described methods (Hara et al., 2012; Markle et al., 2013). Amplicons were generated using

primers that target approximately 300 base pairs of the V1/V2 variable region of the $16 \mathrm{~S}$ rRNA

gene. Each DNA was amplified in triplicate along with a barcode specific negative PCR control.

PCR reactions contained 1X HotMaster Mix (5Prime), $150 \mathrm{nM}$ each PCR primer and template in

a reaction volume of $25 \mu \mathrm{l}$. Cycling conditions were $94{ }^{\circ} \mathrm{C}$ denaturation for 120 seconds followed confirmed using agarose gel electrophoresis. None of the negative PCR controls showed evidence of amplification. PCR products were normalized based on agarose gel densitometry, pooled, lyophilized, purified and concentrated using a DNA Clean and Concentrator Kit (Zymo, Irvine,

110 CA). Pooled amplicons were quantified using Qubit Fluorometer 2.0 (Invitrogen, Carlsbad, CA).

111 The pool was diluted to $4 \mathrm{nM}$ and denatured with $0.2 \mathrm{~N} \mathrm{NaOH}$ at room temperature. The denatured 112 DNA was diluted to $15 \mathrm{pM}$ and spiked with $10 \%$ of the Illumina PhiX control DNA prior to loading 
113 the sequencer. Illumina paired-end sequencing was performed on the MiSeq using a 500 cycle

114 version 2 reagent kit.

115 Analysis of Illumina Paired-end Reads. As previously described, paired-end sequences

116 were sorted by sample via barcodes in the paired reads with a python script (Markle et al., 2013)

117 Sorted paired end sequence data were deposited in the NCBI Short Read Archive under

118 accession number SRP043334. The sorted paired reads were assembled using phrap (Ewing and

119 Green 1998; Ewing et al.1998.). Pairs that did not assemble were discarded. Assembled

120 sequence ends were trimmed over a moving window of 5 nucleotides until average quality met

121 or exceeded 20 . Trimmed sequences with more than 1 ambiguity or shorter than 250 nt were

122 discarded. Potential chimeras identified with Uchime (usearch6.0.203_i86linux32) (Edgar et al.,

123 2011) using the Schloss Silva reference sequences (Schloss \& Westcott, 2011) were removed

124 from subsequent analyses. Assembled sequences were aligned and classified with SINA (1.3.0-

125 r23838)(Pruesse, Peplies \& Glöckner, 2012) using the 479,726 bacterial sequences in Silva

126 115NR (Quast et al., 2013) as reference configured to yield the Silva taxonomy. Sequences with

127 identical taxonomic assignments were grouped into Operational taxonomic units (OTUs). This

128 process generated 4,302,223 sequences for 162 samples (average sequence length: $316 \mathrm{nt}$;

129 average sample size: 26,557 sequences/sample; minimum sample size: 7,527; maximum samples

130 size: 63,105$)$. The median Good's coverage score was $\geq 99.70 \%$ at the rarefaction point of 7,527.

131 The software package Explicet (v2.10.5, www.explicet.org) (Robertson et al., 2013) was used to

132 calculate rarefied values for diversity measurements.

$134(\mathrm{MH})$ beta diversity were calculated in Explicet. MH beta diversity is a measure of similarity

135 between two communities and ranges from 0 (no similarity) to 1 (identical communities). 
136 Principal Coordinate Analysis (PCoA) was used to assess differences at the community level

137 utilizing 1-MH. 1-MH distances were used to show the dissimilarity between with EnzD and

138 without EnzD in PCoA plots instead of MH distances, which are a measure of similarity.

139 Relative abundance (RA) for each genus was calculated by dividing the genera-specific sequence

140 counts by the total number of sequences obtained for each sample. Shannon-H diversity,

141 evenness and richness were compared using a Wilcoxon Signed Rank test. Differences in phyla

142 and genera between EnzD samples versus non-EnzD samples with a median RA of at least 1\%

143 were evaluated using Wilcoxon Signed rank test for paired samples. Benjamini-Hochberg

144 corrections were used to account for multiple comparisons (Benjamini \& Yekutieli, 2001).

145 Spearman correlations were used to assess associations between MH for paired samples and RA

146 of specific genera from the non-digested sample. Analyses were calculated using R version 3.2.4

147 Revised (2016-03-16 r70336).

\section{Results}

Sample Collection. We analyzed 81 airway samples (39 sputum and 42 OP, Figure S1)

151

152

153

154

155

156

157

collected from 63 patients with cystic fibrosis ranging in age from 1.5 to 24 years ( 9 to 24

sputum and 1.5 to $23 \mathrm{OP})$. Half of the subjects were female (51\%), the majority of subjects were non-Hispanic white (89\%) and 52\% of subjects were homozygous F508. The median number of samples collected per subject was 1 and ranged between 1 and 3 samples. Seven samples (9\%) were negative for standard CF pathogens by culture (Figure S2).

Changes in individual organisms. Individual taxa were assessed at the phyla and genera level by sample type (OP and Sputum). For OP samples, we observed a significantly lower RA 
158 for the phyla Bacteroidetes, Fusobacteria and Proteobacteria when EnzD was used. Firmicutes

159 and Actinobacteria both were detected in higher RA with the use of EnzD. For sputum, the RA

160 for Bacteroidetes and Proteobacteria were lower with EnzD (Table 1) consistent with findings in

161 OP samples (Figure 1A).

Genera with a statistically significantly higher RA following EnzD for OP swabs consisted of: Gemella, Streptococcus, Actinomyces, Johnsonella, and Rothia. Genera with a statistically significantly lower RA for OP swabs consisted of: Prevotella, Veillonella, Neisseria, Haemophilus, Leptotrichia, Campylobacter, Fusobacterium, and Granulicatella. For these genera, while the changes are statistically significant, the changes may not be clinically meaningful. The largest changes in RA (>5\%) are seen in Streptococcus, Prevotella, and Veillonella (Table 2). Staphylococcus was the only genus with a significantly higher RA in sputum following EnzD. Genera with a statistically significantly lower RA in sputum consisted of: Veillonella, Granulicatella, Prevotella, and Gemella (Table 2). The largest estimated change in RA for sputum samples is attributed to Staphylococcus (Table 2). Gemella was higher in OP samples with EnzD while it was lower in sputum samples with EnzD (Figure 1B). Haemophilus, Stenotrophomonas, and Achromobacter(Zemanick et al., 2015). All of the samples have low RA for Achromobacter and all of the OP samples had low RA for Pseudomonas and Stenotrophomonas. In the sputum samples, 11 had low amounts of all CF pathogens, 11 were dominated by Staphylococcus, all of which increased with EnzD, and 3 were dominated mostly by Haemophilus. Of the remaining 14 sputum samples, 3 samples were dominated by

179 Stenotrophomonas, 1 increased, 1 decreased and 1 remained unchanged with EnzD. Eleven 180 sputum samples had $>5 \%$ RA Pseudomonas prior to EnzD, the RA increased after EnzD in 6 of 
181 these samples (median 18.7 range $1.1 \%$ to $68.4 \%$ ), in those where RA decreased, the median and

182 range was $-6.1 \%,-1.2 \%$ to $42.0 \%$ (Table 3 ).

resulting in a significantly less even community (Shannon-H, Wilcoxon Signed Rank, $\mathrm{p}<0.01$ ).

For OP samples, the Shannon-H alpha Diversity Index was also lower likely due to higher RA of

Streptococcus with EnzD, although this result did not reach statistical significance (Shannon-H,

Wilcoxon Signed Rank, $\mathrm{p}=0.06$ ). Richness and evenness were also not significantly affected by

EnzD in OP samples. Changes in alpha diversity due to use of EnzD do not necessarily correspond to changes in microbial community composition as measured using beta diversity (Figure S4).

Beta Diversity Changes. There was less impact of EnzD on community composition in sputum compared to OP samples as measured by MH beta diversity values for each sample pair.

The majority of the $\mathrm{MH}$ values (79.5\%) for sputum were greater than 0.8 , whereas only $38.1 \%$ of OP samples were above that level. For the OP swabs the lowest MH was 0.4 for sputum samples the lowest $\mathrm{MH}$ was 0.48 . The distributions between the two sample types were statistically different (MH, Wilcoxon Rank Sum, $\mathrm{p}<0.01$, Figure 2). We further investigated these changes using the MH distance matrix in Principal Coordinate Analysis (PCoA). OP samples changed more consistently in ordination space with EnzD (Figure 3A). The consistent changes observed in the OP samples were largely due to the change in Streptococcus, Gemella, and Rothia (all Gram-positive) as well as Neisseria, Leptotrichia, Prevotella, and Veillonella (Gram-negative); with the samples undergoing EnzD primarily clustered around Streptococcus, Gemella, and Rothia (Figure 3A). Findings here align with previously discussed individual level changes in 
204 genera where Gram-positive bacteria were found in significantly higher RA while Gram-

205 negative bacteria was found in significantly lower RA with EnzD (Table 2). As indicated in

206 Figure 3B, sputum samples showed less impact from EnzD, with a large group of samples

207 having limited changes in $\mathrm{MH}$. A subset of the sputum samples demonstrated large changes in

208 MH with EnzD, but with no consistent direction of change as was seen in the OP samples

209 (Figure 3B). Sputum appears to be more heterogeneous compared to OP samples. Sputum was

210 primarily influenced by enhanced extraction of Staphylococcus, Veillonella, and Streptococcus

211 (Figure 3B). This result is consistent with Table 2 where Staphylococcus and Veillonella are

212 shown to have a larger change than Streptococcus.

214 a single phylum or genus whose relative abundance was associated with MH (Figure S5).

215 Combination of Gram-positive bacteria was calculated using the summation of (Actinobacteria +

216 Firmicutes (-Veillonella)). For the OP samples with large changes in their communities with

217 EnzD, the relative abundance of Gram-positive organisms was lower compared to those samples

218 with limited changes in their communities (Figure 4). For all the OP samples, the relative

219 abundance of Gram-positive organisms was higher with EnzD. There was an association

220 between change in relative abundance of Gram-positive organisms with EnzD and MH values

221 for the pairs (Spearman's correlation, $r=-0.60 ; \mathrm{p}<0.01$; Figure 4B).

\section{Discussion}

223 In this work, we demonstrate that EnzD of airway samples changes the bacterial

224 community detected by sequencing primarily due to increased detection of organisms with a

225 Gram-positive cell wall structure. From the biplots (Figure 3A) we can see that for OP samples

226 there is a clear shift in microbial composition between those without EnzD and those with EnzD. 
227 With EnzD the OP samples are highly clustered around Gemella, Rothia, and Streptococcus

228 whereas those without EnzD are dispersed around Neisseria, Prevotella and Veillonella. These

229 observations are consistent with cell wall structure; we would predict that given their rigid cell

230 wall Gram-positive bacteria would drive changes in community composition for those samples

231 with EnzD. Streptococcus, Gemella, and Rothia explain a large amount of the variability in the

232 OP samples. Streptococcus and Staphylococcus are Gram-positive taxa explaining a large

233 amount of the variability in the sputum samples. Among the Gram-negative taxa explaining a

234 large amount of variability in $\mathrm{OP}$ and sputum samples are Neisseria, Veillonella, and Prevotella

235 due to decreasing relative abundance with EnzD. Granulicatella, a member of the Firmicutes had

236 decreased RA with EnzD, which was not consistent with other Gram positive taxa.

237 Granulicatella is reported to have pleomorphic and variable Gram stain characteristics, which

238 may explain this observation(Bottone et al., 1995; Christensen \& Facklam, 2001).

239 Difference in OP and sputum samples can be due to differences in the microbial

240 communities which may be related to multiple factors including anatomic location, inter

241 individual variability and disease severity. The samples used in this study were collected for

242 clinical evaluation of airway infection, although separate evaluation for sample adequacy was

243 not performed, these samples are reflective of what is being used to make clinical decisions. The

244 patients that were not capable of expectorating sputum tend to be younger with presumably less

245 lung disease (i.e., fewer pathogens). There are also differences between upper and lower airways

246 (Zemanick et al., 2015) that could also impact the community composition observed.

Willner et al. demonstrated that genera detection varied significantly between 5

commonly used extraction methods with Staphylococcus being detected with varying efficiency

249 (Willner et al., 2012). Support for these findings showed the largest difference in microbial 
250 proportions between two DNA extraction methods (Norgen and Qiagen) was found in

251 Staphylococcus (Pérez-Losada et al., 2016). Consistent with previous findings, EnzD revealed a

252 higher RA of Staphylococcus in OP samples. Further, when evaluated in both OP swab and

253 sputum samples from pediatric subjects with CF, variations in detection of Staphylococcus were

254 observed (Johnson et al., 2016). Total RA for Staphylococcus in sputum samples was higher

255 overall, but had less of a change before and after EnzD (Johnson et al., 2016). Zhao et al. looked

256 at sputum and saw an increase in Staphylococcus, which we corroborate (Zhao et al., 2012). We

257 also found a higher RA of Streptococcus in OP samples. Johnson et al. found that for OP

258 samples DNA concentration consistently increased with EnzD. This indicates that in addition to

259 observing changes in RA, EnzD probably better represents the absolute amounts of genera

260 present in OP samples.

Limitations. There are certain limitations to our study. First, only two airway sample

262

263

264

265

266

267

268

269

270

271 types collected from subjects with CF were evaluated, thus limiting more general inferences regarding the effects of EnzD in other samples types. However, this study does include OP samples, which have not previously been evaluated and represents an important sample type in early CF. The patient's clinical status at the time of sample collection is unknown, this hampers our ability to determine whether these samples are reflective of both clinical stability or during a pulmonary exacerbation. However, because paired samples were used to assess the effect of EnzD, the difference for each pair in not confounded by clinical status. Because only a single replicate with and without EnzD was evaluated, we are unable to compare effects due to EnzD versus technical variability. However, the observation of the consistent differences for the majority of the sample pairs provides some evidence that the change is due to EnzD rather than 
272 technical variability. In our study we considered beta-diversity values of 0.8 or greater to be

273 within the limits of change due to biological variability.

274 The utility of EnzD is difficult to predict due to the strong influence of the community

275 composition in any given sample on the results. There is a compelling argument to utilize EnzD

276 in studies that rely on OP swabs as previous studies have shown increased sensitivity for

277 detection of Staphylococcus. While the argument is less compelling for sputum, EnzD should be

278 applied consistently. Other sample types would require evaluation to determine how much

279 impact is observed. However, increasing the RA of any group makes it harder to identify minor

280 components in the community. EnzD could negatively impact the ability to identify low

281 abundance Gram-negative organisms as seen through the decrease in Gram-negative genera

282 shown in Table 2. This includes many important pathogens in CF (e.g. Pseudomonas) and should

283 be considered carefully.

\section{Conclusions}

285

286

287

288

289

290

291

292

293
In summary, we found that the use of EnzD on airway samples prior to DNA extraction and sequencing alters microbiome community composition results, likely due to improved detection of gram-positive bacterial taxa (e.g. Streptococcus and Staphylococcus). The use of EnzD appears particularly important for analysis of OP swab samples. EnzD use with sputum samples may be less critical and has the potential to decrease sensitivity for low abundance taxa. Our findings highlight the need for a consistent approach to airway sample processing and analysis, and suggest that EnzD should be applied routinely in studies using OP swabs and studies requiring sensitive detection of Staphylococcus and Streptococcus. 
294

295

296

297

298

299

300

301

302

303

304

305

306

307

308

309

310

311

312

313

314

\section{References}

Armstrong DS., Grimwood K., Carlin JB., Carzino R., Olinsky A., Phelan PD. 1996.

Bronchoalveolar Lavage or Oropharyngeal Cultures to Identify Lower Respiratory

Pathogens in Infants With Cystic Fibrosis. Pediatric Pulmonology Pedlatr Pulmonol 21.

Benjamini Y., Yekutieli D. 2001. THE CONTROL OF THE FALSE DISCOVERY RATE IN

MULTIPLE TESTING UNDER DEPENDENCY. The Annals of Statistics 29:1165-1188.

Bottone EJ., Thomas CA., Lindquist D., Janda JM. 1995. Difficulties encountered in identification of a nutritionally deficient streptococcus on the basis of its failure to revert to streptococcal morphology. Journal of clinical microbiology 33:1022-4.

Browder HP., Zygmunt WA., Young JR., Tavormina PA. 1965. Lysostaphin: Enzymatic mode of action. Biochemical and Biophysical Research Communications 19:383-389. DOI: 10.1016/0006-291X(65)90473-0.

Burns JL., Emerson J., Stapp JR., Yim DL., Krzewinski J., Louden L., Ramsey BW., Clausen CR. 1998. Microbiology of sputum from patients at cystic fibrosis centers in the United States. Clinical infectious diseases : an official publication of the Infectious Diseases Society of America 27:158-63.

Christensen JJ., Facklam RR. 2001. Granulicatella and Abiotrophia species from human clinical specimens. Journal of clinical microbiology 39:3520-3. DOI: 10.1128/JCM.39.10.35203523.2001.

Cummings LA., Kurosawa K., Hoogestraat DR., SenGupta DJ., Candra F., Doyle M., Thielges S., Land TA., Rosenthal CA., Hoffman NG., Salipante SJ., Cookson BT. 2016. Clinical 
Edgar RC., Haas BJ., Clemente JC., Quince C., Knight R. 2011. UCHIME improves sensitivity and speed of chimera detection. Bioinformatics (Oxford, England) 27:2194-200. DOI: 10.1093/bioinformatics/btr381.

Ewing B., Green P. 1998. Base-Calling of Automated Sequencer Traces Using Phred. II. Error Probabilities. Genome Research 8:186-194. DOI: 10.1101/gr.8.3.186.

Gradisar H., Friedrich J., Krizaj I., Jerala R. 2005. Similarities and specificities of fungal keratinolytic proteases: comparison of keratinases of Paecilomyces marquandii and Doratomyces microsporus to some known proteases. Applied and environmental microbiology 71:3420-6. DOI: 10.1128/AEM.71.7.3420-3426.2005.

Hara N., Alkanani AK., Ir D., Robertson CE., Wagner BD., Frank DN., Zipris D. 2012. Prevention of Virus-Induced Type 1 Diabetes with Antibiotic Therapy. The Journal of

Heijerman H. 2005. Infection and inflammation in cystic fibrosis: a short review. Journal of Immunology 189:3805-3814. DOI: 10.4049/jimmunol.1201257.

Hoppe JE., Towler E., Wagner BD., Accurso FJ., Sagel SD., Zemanick ET. 2015. Sputum induction improves detection of pathogens in children with cystic fibrosis. Pediatric pulmonology 50:638-46. DOI: 10.1002/ppul.23150. 
336

337

Molecular identification of Staphylococcus aureus in airway samples from children with cystic fibrosis. PLoS ONE 11:1-12. DOI: 10.1371/journal.pone.0147643.

LiPuma JJ. 2010. The Changing Microbial Epidemiology in Cystic Fibrosis. Clinical Microbiology Reviews 23:299-323. DOI: 10.1128/CMR.00068-09.

Lozupone CA., Stombaugh J., Gonzalez A., Ackermann G., Wendel D., Vazquez-Baeza Y., Jansson JK., Gordon JI., Knight R. 2013. Meta-analyses of studies of the human microbiota. Genome Research 23:1704-1714. DOI: 10.1101/gr.151803.112.

Markle JGM., Frank DN., Mortin-Toth S., Robertson CE., Feazel LM., Rolle-Kampczyk U., von Bergen M., McCoy KD., Macpherson AJ., Danska JS. 2013. Sex differences in the gut microbiome drive hormone-dependent regulation of autoimmunity. Science (New York, N.Y.) 339:1084-8. DOI: 10.1126/science.1233521.

Pérez-Losada M., Crandall K., Freishtat RJ., Pé Rez-Losada M., Crandall KA. 2016. Comparison of two commercial DNA extraction kits for the analysis of nasopharyngeal bacterial communities APA Citation Comparison of two commercial DNA extraction kits for the analysis of nasopharyngeal bacterial communities. AIMS Microbiology 2:108-119. DOI: 10.3934/microbiol.2016.2.108.

Pruesse E., Peplies J., Glöckner FO. 2012. SINA: accurate high-throughput multiple sequence alignment of ribosomal RNA genes. Bioinformatics (Oxford, England) 28:1823-9. DOI: 10.1093/bioinformatics/bts252.

Quast C., Pruesse E., Yilmaz P., Gerken J., Schweer T., Yarza P., Peplies J., Glöckner FO. 2013. The SILVA ribosomal RNA gene database project: improved data processing and webbased tools. Nucleic acids research 41:D590-6. DOI: 10.1093/nar/gks1219. 
358 Robertson CE., Harris JK., Wagner BD., Granger D., Browne K., Tatem B., Feazel LM., Park

359 K., Pace NR., Frank DN. 2013. Explicet: graphical user interface software for metadata360 driven management, analysis and visualization of microbiome data. Bioinformatics (Oxford,

361 England) 29:3100-1. DOI: 10.1093/bioinformatics/btt526.

362 Salazar O., Asenjo JA. 2007. Enzymatic lysis of microbial cells. DOI: 10.1007/s10529-007-

$363 \quad 9345-2$.

364

365

366

367

368

369

370

371

372

373

374

375

376

377

378

379

Schindler CA., Schuhardt VT. 1964. Lysostaphin: A New Bacteriolytic Agent for the Staphylococcus. Proceedings of the National Academy of Sciences of the United States of America 51:414-421. DOI: 10.1073/pnas.51.3.414.

Schloss PD., Westcott SL. 2011. Assessing and Improving Methods Used in Operational Taxonomic Unit-Based Approaches for 16S rRNA Gene Sequence Analysis. Applied and Environmental Microbiology 77:3219-3226. DOI: 10.1128/AEM.02810-10.

Trayer HR., Buckley CE. 1970. Molecular properties of lysostaphin, a bacteriolytic agent specific for Staphylococcus aureus. The Journal of biological chemistry 245:4842-6.

Willner D., Daly J., Whiley D., Grimwood K., Wainwright CE., Hugenholtz P., Tringe S., Hugenholtz P. 2012. Comparison of DNA Extraction Methods for Microbial Community Profiling with an Application to Pediatric Bronchoalveolar Lavage Samples. PLoS ONE 7:e34605. DOI: 10.1371/journal.pone.0034605.

Wolter DJ., Emerson JC., McNamara S., Buccat AM., Qin X., Cochrane E., Houston LS., Rogers GB., Marsh P., Prehar K., Pope CE., Blackledge M., Deziel E., Bruce KD., Ramsey BW., Gibson RL., Burns JL., Hoffman LR. 2013. Staphylococcus aureus Small-Colony Variants Are Independently Associated With Worse Lung Disease in Children With Cystic Fibrosis. 
381 Yuan S., Cohen DB., Ravel J., Abdo Z., Forney LJ. 2012b. Evaluation of Methods for the 382 Extraction and Purification of DNA from the Human Microbiome. PLoS ONE 7:e33865. 383 DOI: 10.1371/journal.pone.0033865.

384 Zemanick ET., Wagner BD., Harris JK., Wagener JS., Accurso FJ., Sagel SD. 2010. Pulmonary 385 exacerbations in cystic fibrosis with negative bacterial cultures. Pediatric pulmonology $386 \quad$ 45:569-77. DOI: 10.1002/ppul.21221.

Zemanick ET., Wagner BD., Robertson CE., Stevens MJ., Szefler SJ., Accurso FJ., Sagel SD., 388 Harris JK. 2015. Assessment of Airway Microbiota and Inflammation in Cystic Fibrosis 389 Using Multiple Sampling Methods. Annals of the American Thoracic Society 12:221-229. 390 DOI: 10.1513/AnnalsATS.201407-310OC. 2012. Impact of enhanced Staphylococcus DNA extraction on microbial community measures in cystic fibrosis sputum. PloS one 7:e33127. DOI: 10.1371/journal.pone.0033127. 


\section{Table $\mathbf{1}$ (on next page)}

Table 1

Median relative abundance in EnzD and Non-EnzD groups; estimated change (EnzD - NonEnzD) in median RA by Phyla. 


\begin{tabular}{|c|c|c|c|c|c|}
\hline \multicolumn{6}{|c|}{ OP } \\
\hline Phyla & EnzD \% & $\begin{array}{l}\text { Non-EnzD } \\
\%\end{array}$ & $\begin{array}{l}\text { Estimated change in RA } \\
\%\end{array}$ & P-Value & $\begin{array}{l}\text { Corrected } \\
\text { p-Value }\end{array}$ \\
\hline Actinobacteria & 5.60 & 1.25 & 3.79 & $<0.01$ & $<0.01$ \\
\hline Bacteroidetes & 7.80 & 19.35 & -12.73 & $<0.01$ & $<0.01$ \\
\hline Firmicutes & 68.31 & 48.32 & 16.78 & $<0.01$ & $<0.01$ \\
\hline Fusobacteria & 3.50 & 8.83 & -1.97 & $<0.01$ & $<0.01$ \\
\hline Proteobacteria & 2.88 & 7.71 & -5.52 & $<0.01$ & $<0.01$ \\
\hline \multicolumn{6}{|c|}{ Sputum } \\
\hline Phyla & EnzD \% & $\begin{array}{r}\text { Non-EnzD } \\
\%\end{array}$ & $\begin{array}{r}\text { Estimated change in } \mathrm{RA} \\
\%\end{array}$ & P-Value & $\begin{array}{r}\text { Corrected } \\
\text { p-Value }\end{array}$ \\
\hline Actinobacteria & 0.03 & 0.01 & 1.12 & 0.06 & 0.06 \\
\hline Bacteroidetes & 0.01 & 0.06 & -3.52 & $<0.01$ & $<0.01$ \\
\hline Firmicutes & 0.84 & 0.72 & 4.77 & 0.08 & 0.08 \\
\hline Proteobacteria & 0.01 & 0.02 & -1.36 & $<0.01$ & $<0.01$ \\
\hline
\end{tabular}




\section{Table 2 (on next page)}

Table 2

Median relative abundance in EnzD and Non-EnzD groups; estimated change (EnzD- NonEnzD); in Median RA by Genera. 


\begin{tabular}{|c|c|c|c|c|c|}
\hline \multicolumn{6}{|c|}{ OP } \\
\hline Genera & EnzD \% & $\begin{array}{l}\text { Non-EnzD } \\
\%\end{array}$ & $\begin{array}{l}\text { Estimated change in RA } \\
\%\end{array}$ & P-Value & $\begin{array}{l}\text { Corrected } \\
\text { p-Value }\end{array}$ \\
\hline Streptococcus & 36.51 & 14.81 & 21.75 & $<0.01$ & $<0.01$ \\
\hline Prevotella & 6.62 & 16.20 & -10.90 & $<0.01$ & $<0.01$ \\
\hline Veillonella & 9.27 & 19.22 & -7.86 & $<0.01$ & $<0.01$ \\
\hline Neisseria & 0.72 & 1.94 & -3.62 & $<0.01$ & $<0.01$ \\
\hline Actinomyces & 2.93 & 0.73 & 2.50 & $<0.01$ & $<0.01$ \\
\hline Granulicatella & 1.80 & 2.44 & -0.62 & $<0.01$ & $<0.01$ \\
\hline Gemella & 1.89 & 0.64 & 1.23 & $<0.01$ & $<0.01$ \\
\hline Leptotrichia & 1.16 & 3.79 & -1.44 & $<0.01$ & $<0.01$ \\
\hline Fusobacterium & 1.47 & 3.08 & -0.56 & $<0.01$ & $<0.01$ \\
\hline Rothia & 1.70 & 0.27 & 1.25 & $<0.01$ & $<0.01$ \\
\hline Haemophilus & 0.45 & 1.69 & -0.91 & $<0.01$ & $<0.01$ \\
\hline Johnsonella & 0.97 & 0.40 & 1.19 & $<0.01$ & $<0.01$ \\
\hline Campylobacter & 0.50 & 0.75 & -0.25 & $<0.01$ & $<0.01$ \\
\hline \multicolumn{6}{|c|}{ Sputum } \\
\hline Genera & EnzD \% & $\begin{array}{r}\text { Non-EnzD } \\
\%\end{array}$ & $\begin{array}{r}\text { Estimated change in } \mathrm{RA} \\
\%\end{array}$ & P-Value & $\begin{array}{r}\text { Corrected } \\
\text { p-Value }\end{array}$ \\
\hline Streptococcus & 27.85 & 22.48 & 1.33 & 0.50 & 0.50 \\
\hline Staphylococcus & 1.39 & 0.62 & 5.47 & $<0.01$ & $<0.01$ \\
\hline Prevotella & 0.88 & 3.54 & -1.91 & $<0.01$ & $<0.01$ \\
\hline Veillonella & 1.09 & 6.01 & -3.55 & $<0.01$ & $<0.01$ \\
\hline Granulicatella & 0.53 & 1.77 & -1.30 & $<0.01$ & $<0.01$ \\
\hline Gemella & 0.53 & 1.68 & -0.62 & $<0.01$ & 0.02 \\
\hline Rothia & 0.84 & 0.57 & 0.21 & 0.24 & 0.27 \\
\hline
\end{tabular}




\section{Table 3(on next page)}

Table 3

Displays the RA for CF pathogens detected in each sample. Samples are highlighted when the difference in relative abundance was at least 1\%, red for increase with EnzD and blue with a decrease. The grey rows indicate the median ( $\min , \max$ ) for the difference in relative abundance for the highlighted samples. 


\begin{tabular}{|c|c|c|c|c|c|c|c|c|c|c|}
\hline & \multicolumn{2}{|c|}{ Staphylococcus } & \multicolumn{2}{|c|}{ Achromobacter } & \multicolumn{2}{|c|}{ Haemophilus } & \multicolumn{2}{|c|}{ Stenotrophomonas } & \multicolumn{2}{|c|}{ Pseudomonas } \\
\hline & $\begin{array}{l}\text { Non } \\
\text { EnzD }\end{array}$ & EnzD & $\begin{array}{l}\text { Non } \\
\text { EnzD }\end{array}$ & EnzD & $\begin{array}{l}\text { Non } \\
\text { EnzD }\end{array}$ & EnzD & $\begin{array}{l}\text { Non } \\
\text { EnzD }\end{array}$ & EnzD & $\begin{array}{l}\text { Non } \\
\text { EnzD }\end{array}$ & EnzD \\
\hline \multicolumn{11}{|c|}{ OP } \\
\hline OP1 & 0 & $0.01 \%$ & 0 & 0 & $1.63 \%$ & $0.34 \%$ & 0 & 0 & 0 & 0 \\
\hline OP2 & 0 & $0.08 \%$ & 0 & 0 & $4.03 \%$ & $1.56 \%$ & 0 & 0 & 0 & 0 \\
\hline OP3 & $0.01 \%$ & $0.07 \%$ & 0 & 0 & $0.04 \%$ & 0 & 0 & 0 & 0 & 0 \\
\hline OP4 & $0.01 \%$ & $0.03 \%$ & 0 & 0 & $1.96 \%$ & $0.56 \%$ & 0 & 0 & 0 & 0 \\
\hline OP5 & $0.01 \%$ & $0.04 \%$ & 0 & 0 & $2.65 \%$ & $0.83 \%$ & 0 & 0 & 0 & 0 \\
\hline OP6 & 0 & $0.01 \%$ & 0 & 0 & $0.00 \%$ & $0.01 \%$ & 0 & $0.01 \%$ & 0 & 0 \\
\hline OP7 & 0 & 0 & 0 & 0 & $5.71 \%$ & $1.46 \%$ & 0 & 0 & 0 & 0 \\
\hline OP8 & $0.15 \%$ & $1.13 \%$ & 0 & 0 & $2.05 \%$ & $0.65 \%$ & 0 & 0 & 0 & 0 \\
\hline OP9 & 0 & $0.02 \%$ & 0 & 0 & $15.12 \%$ & $7.11 \%$ & 0 & 0 & 0 & 0 \\
\hline OP10 & 0 & $0.04 \%$ & 0 & 0 & $0.25 \%$ & $0.08 \%$ & 0 & 0 & 0 & 0 \\
\hline OP11 & $0.01 \%$ & $0.03 \%$ & 0 & 0 & $5.86 \%$ & $2.03 \%$ & 0 & 0 & 0 & 0 \\
\hline OP12 & $0.02 \%$ & $0.03 \%$ & 0 & 0 & $0.21 \%$ & $0.11 \%$ & 0 & 0 & $0.01 \%$ & $0.02 \%$ \\
\hline OP13 & $0.08 \%$ & $0.30 \%$ & 0 & 0 & $1.19 \%$ & $0.22 \%$ & $0.01 \%$ & 0 & 0 & 0 \\
\hline OP14 & $0.06 \%$ & $0.37 \%$ & 0 & 0 & $2.41 \%$ & $1.43 \%$ & 0 & 0 & $0.03 \%$ & 0 \\
\hline OP15 & $0.07 \%$ & $1.02 \%$ & 0 & 0 & $0.03 \%$ & $0.04 \%$ & 0 & 0 & $0.01 \%$ & $0.01 \%$ \\
\hline OP16 & $0.04 \%$ & $0.05 \%$ & 0 & 0 & $0.09 \%$ & $0.02 \%$ & 0 & 0 & 0 & 0 \\
\hline OP17 & $0.01 \%$ & $0.08 \%$ & 0 & 0 & $0.57 \%$ & $0.31 \%$ & 0 & 0 & 0 & 0 \\
\hline OP18 & $0.01 \%$ & $0.01 \%$ & 0 & 0 & $3.49 \%$ & $1.87 \%$ & 0 & 0 & $0.01 \%$ & $0.01 \%$ \\
\hline OP19 & $0.01 \%$ & $0.57 \%$ & 0 & 0 & $1.91 \%$ & $0.57 \%$ & 0 & 0 & 0 & $0.01 \%$ \\
\hline OP20 & 0 & 0 & 0 & 0 & $3.45 \%$ & $1.79 \%$ & 0 & 0 & 0 & 0 \\
\hline OP21 & $0.02 \%$ & $0.10 \%$ & 0 & 0 & $0.40 \%$ & $0.18 \%$ & 0 & 0 & $0.01 \%$ & $0.02 \%$ \\
\hline OP22 & 0 & 0 & 0 & 0 & $0.35 \%$ & $0.05 \%$ & 0 & 0 & $0.01 \%$ & $0.05 \%$ \\
\hline OP23 & $0.07 \%$ & $0.18 \%$ & 0 & 0 & $11.95 \%$ & $7.84 \%$ & $0.01 \%$ & 0 & 0 & $0.01 \%$ \\
\hline OP24 & 0 & 0 & 0 & 0 & $0.17 \%$ & $0.03 \%$ & 0 & 0 & 0 & 0 \\
\hline OP25 & $0.01 \%$ & $0.01 \%$ & 0 & 0 & $0.23 \%$ & $0.07 \%$ & $0.01 \%$ & $0.01 \%$ & $0.02 \%$ & $0.01 \%$ \\
\hline OP26 & $2.17 \%$ & $3.92 \%$ & 0 & 0 & $0.45 \%$ & $0.04 \%$ & 0 & 0 & $0.01 \%$ & 0 \\
\hline OP27 & $0.11 \%$ & $0.16 \%$ & 0 & 0 & 0 & 0 & 0 & 0 & 0 & $0.01 \%$ \\
\hline OP28 & $0.05 \%$ & 0 & 0 & 0 & $2.44 \%$ & $1.82 \%$ & 0 & 0 & 0 & 0 \\
\hline OP29 & 0 & 0 & 0 & 0 & $1.75 \%$ & $0.67 \%$ & $0.05 \%$ & $0.03 \%$ & 0 & 0 \\
\hline OP30 & $0.01 \%$ & $0.03 \%$ & 0 & 0 & $40.38 \%$ & $30.81 \%$ & 0 & 0 & 0 & 0 \\
\hline OP31 & 0 & 0 & 0 & 0 & $0.61 \%$ & $0.14 \%$ & 0 & 0 & 0 & 0 \\
\hline OP32 & $0.01 \%$ & $0.01 \%$ & 0 & 0 & $3.40 \%$ & $1.09 \%$ & 0 & 0 & $0.07 \%$ & $0.06 \%$ \\
\hline OP33 & 0 & 0 & 0 & 0 & $0.30 \%$ & $0.04 \%$ & 0 & 0 & 0 & 0 \\
\hline OP34 & $3.34 \%$ & $14.25 \%$ & 0 & 0 & $2.01 \%$ & $1.19 \%$ & 0 & 0 & 0 & 0 \\
\hline OP35 & $0.05 \%$ & $0.08 \%$ & 0 & 0 & $4.44 \%$ & $4.27 \%$ & 0 & 0 & 0 & 0 \\
\hline OP36 & $0.02 \%$ & $0.22 \%$ & 0 & 0 & $0.03 \%$ & $0.01 \%$ & 0 & 0 & 0 & 0 \\
\hline OP37 & $0.11 \%$ & $0.19 \%$ & 0 & 0 & $2.06 \%$ & $0.58 \%$ & 0 & 0 & 0 & 0 \\
\hline OP38 & $0.01 \%$ & $0.04 \%$ & 0 & 0 & $3.68 \%$ & $1.54 \%$ & 0 & 0 & 0 & 0 \\
\hline OP39 & $0.06 \%$ & $0.07 \%$ & 0 & 0 & 0 & 0 & 0 & 0 & $0.01 \%$ & 0 \\
\hline OP40 & $0.13 \%$ & $0.11 \%$ & 0 & 0 & $0.01 \%$ & 0 & 0 & 0 & $0.01 \%$ & $0.02 \%$ \\
\hline
\end{tabular}




\begin{tabular}{|c|c|c|c|c|c|c|c|c|c|c|}
\hline OP41 & $0.05 \%$ & $0.03 \%$ & 0 & 0 & $1.91 \%$ & $1.20 \%$ & 0 & 0 & 0 & 0 \\
\hline OP42 & $0.01 \%$ & $0.01 \%$ & 0 & 0 & $0.05 \%$ & $0.01 \%$ & 0 & 0 & $0.02 \%$ & $0.01 \%$ \\
\hline & \multicolumn{2}{|c|}{$6.3(1.8,10.9)$} & \multicolumn{2}{|c|}{--} & \multicolumn{2}{|c|}{$-1.8(-1.1,-9.6)$} & \multicolumn{2}{|c|}{--} & \multicolumn{2}{|c|}{--} \\
\hline & \multicolumn{2}{|c|}{ Staphylococcus } & \multicolumn{2}{|c|}{ Achromobacter } & \multicolumn{2}{|c|}{ Haemophilus } & \multicolumn{2}{|c|}{ Stenotrophomonas } & \multicolumn{2}{|c|}{ Pseudomonas } \\
\hline & $\begin{array}{l}\text { Non } \\
\text { EnzD }\end{array}$ & EnzD & $\begin{array}{l}\text { Non } \\
\text { EnzD }\end{array}$ & EnzD & $\begin{array}{l}\text { Non } \\
\text { EnzD }\end{array}$ & EnzD & $\begin{array}{r}\text { Non } \\
\text { EnzD }\end{array}$ & EnzD & $\begin{array}{l}\text { Non } \\
\text { EnzD }\end{array}$ & EnzD \\
\hline \multicolumn{11}{|c|}{ Sputum } \\
\hline ES1 & $2.24 \%$ & $1.99 \%$ & 0 & 0 & 0 & 0 & 0 & 0 & $0.66 \%$ & $0.51 \%$ \\
\hline ES2 & $0.01 \%$ & $0.05 \%$ & 0 & 0 & $2.89 \%$ & $2.79 \%$ & 0 & 0 & $39.12 \%$ & $79.91 \%$ \\
\hline ES3 & 0 & 0 & 0 & 0 & $8.77 \%$ & $0.83 \%$ & 0 & 0 & 0 & $0.01 \%$ \\
\hline ES4 & $0.05 \%$ & $0.12 \%$ & 0 & 0 & $9.52 \%$ & $6.47 \%$ & 0 & 0 & $1.27 \%$ & $1.02 \%$ \\
\hline ES5 & $34.61 \%$ & $72.13 \%$ & 0 & 0 & 0 & 0 & 0 & 0 & 0 & $0.01 \%$ \\
\hline ES6 & $43.91 \%$ & $84.67 \%$ & 0 & 0 & $0.01 \%$ & 0 & 0 & 0 & $5.04 \%$ & $1.39 \%$ \\
\hline ES7 & $89.38 \%$ & $97.47 \%$ & 0 & 0 & 0 & 0 & $0.07 \%$ & $0.02 \%$ & $0.01 \%$ & 0 \\
\hline ES8 & $16.72 \%$ & $32.21 \%$ & 0 & 0 & 0 & 0 & 0 & 0 & $52.01 \%$ & $9.96 \%$ \\
\hline ES9 & $0.49 \%$ & $8.24 \%$ & 0 & 0 & $1.53 \%$ & $0.71 \%$ & 0 & 0 & $26.46 \%$ & $33.92 \%$ \\
\hline ES10 & $0.20 \%$ & $0.35 \%$ & 0 & 0 & $0.54 \%$ & $0.09 \%$ & 0 & 0 & 0 & $0.01 \%$ \\
\hline ES11 & $31.47 \%$ & $96.31 \%$ & 0 & 0 & $4.11 \%$ & $0.23 \%$ & $0.14 \%$ & $0.02 \%$ & $0.01 \%$ & 0 \\
\hline ES12 & $0.04 \%$ & $0.04 \%$ & 0 & 0 & 0 & 0 & 0 & 0 & $10.08 \%$ & $3.89 \%$ \\
\hline ES13 & $26.05 \%$ & $11.45 \%$ & 0 & 0 & $1.08 \%$ & $0.72 \%$ & 0 & 0 & $31.81 \%$ & $58.28 \%$ \\
\hline ES14 & $0.06 \%$ & $0.13 \%$ & 0 & 0 & 0 & 0 & 0 & 0 & $0.02 \%$ & $0.03 \%$ \\
\hline ES15 & $16.08 \%$ & $29.44 \%$ & 0 & 0 & $11.39 \%$ & $7.50 \%$ & 0 & 0 & $13.04 \%$ & $14.15 \%$ \\
\hline ES16 & $1.48 \%$ & $7.26 \%$ & 0 & 0 & $2.26 \%$ & $1.25 \%$ & 0 & 0 & $52.82 \%$ & $63.76 \%$ \\
\hline ES17 & $67.38 \%$ & $86.70 \%$ & 0 & 0 & $0.17 \%$ & $0.02 \%$ & 0 & 0 & $1.69 \%$ & $0.88 \%$ \\
\hline ES18 & $0.03 \%$ & $0.05 \%$ & 0 & 0 & 0 & 0 & 0 & 0 & $0.01 \%$ & $0.03 \%$ \\
\hline ES19 & $0.62 \%$ & $1.39 \%$ & 0 & 0 & $6.75 \%$ & $2.09 \%$ & $11.95 \%$ & $12.85 \%$ & $0.01 \%$ & 0 \\
\hline ES20 & $88.34 \%$ & $98.55 \%$ & 0 & 0 & 0 & 0 & 0 & 0 & $0.01 \%$ & 0 \\
\hline ES21 & $0.01 \%$ & $0.01 \%$ & 0 & 0 & 0 & 0 & $56.44 \%$ & $37.37 \%$ & 0 & 0 \\
\hline ES22 & $1.08 \%$ & $1.31 \%$ & 0 & 0 & $6.74 \%$ & $8.94 \%$ & 0 & 0 & 0 & 0 \\
\hline ES23 & $0.07 \%$ & $0.34 \%$ & 0 & 0 & $0.01 \%$ & 0 & 0 & 0 & $0.16 \%$ & $0.23 \%$ \\
\hline ES24 & $0.07 \%$ & $0.15 \%$ & 0 & 0 & $0.60 \%$ & $0.28 \%$ & $0.01 \%$ & 0 & $87.24 \%$ & $81.12 \%$ \\
\hline ES25 & 0 & 0 & 0 & 0 & $1.18 \%$ & $0.41 \%$ & $2.41 \%$ & $1.51 \%$ & 0 & 0 \\
\hline ES26 & $41.52 \%$ & $87.96 \%$ & 0 & 0 & $5.60 \%$ & $0.64 \%$ & 0 & 0 & 0 & 0 \\
\hline ES27 & $64.41 \%$ & $97.56 \%$ & 0 & 0 & $0.43 \%$ & $0.02 \%$ & $2.93 \%$ & $0.11 \%$ & 0 & 0 \\
\hline ES28 & $0.02 \%$ & $0.04 \%$ & 0 & 0 & $0.01 \%$ & 0 & $0.01 \%$ & $0.04 \%$ & $0.02 \%$ & $0.01 \%$ \\
\hline ES29 & $73.98 \%$ & $98.00 \%$ & $0.01 \%$ & 0 & 0 & 0 & 0 & 0 & $0.05 \%$ & $0.01 \%$ \\
\hline ES30 & $72.00 \%$ & $87.13 \%$ & 0 & 0 & 0 & 0 & 0 & 0 & $0.18 \%$ & $0.14 \%$ \\
\hline ES31 & $90.32 \%$ & $96.74 \%$ & 0 & 0 & 0 & 0 & $0.01 \%$ & $0.01 \%$ & 0 & 0 \\
\hline ES32 & $0.02 \%$ & $0.01 \%$ & 0 & 0 & $0.02 \%$ & 0 & $33.62 \%$ & $57.14 \%$ & $0.07 \%$ & $0.04 \%$ \\
\hline ES33 & $49.36 \%$ & $78.78 \%$ & 0 & 0 & 0 & 0 & $0.07 \%$ & 0 & $0.03 \%$ & $0.06 \%$ \\
\hline ES34 & $0.05 \%$ & $0.15 \%$ & 0 & 0 & 0 & 0 & 0 & 0 & 0 & $0.03 \%$ \\
\hline ES35 & $1.84 \%$ & $7.29 \%$ & 0 & 0 & $0.66 \%$ & $0.40 \%$ & $0.17 \%$ & $0.10 \%$ & $22.94 \%$ & $21.79 \%$ \\
\hline ES36 & $0.30 \%$ & $0.16 \%$ & 0 & 0 & $0.32 \%$ & $0.02 \%$ & $0.01 \%$ & $0.02 \%$ & $28.07 \%$ & $96.42 \%$ \\
\hline ES37 & 0 & $0.02 \%$ & 0 & 0 & $1.90 \%$ & $1.22 \%$ & 0 & 0 & $0.18 \%$ & $0.31 \%$ \\
\hline
\end{tabular}




\begin{tabular}{|c|c|c|c|c|c|c|c|c|c|c|}
\hline ES38 & $0.16 \%$ & $0.53 \%$ & $0.08 \%$ & $0.10 \%$ & $0.05 \%$ & $0.10 \%$ & 0 & 0 & $0.01 \%$ & $0.03 \%$ \\
\hline $\mathrm{ES} 3$ & $0.01 \%$ & 0 & 0 & 0 & 0 & 0 & 0 & 0 & 0 & $0.01 \%$ \\
\hline & $15.3(-14$ & , 64.8) & & & $-3.9(-7$ & $9,2.2)$ & $-2.8(-19$ & & $1.1(-42$ & 68.4) \\
\hline
\end{tabular}

1 


\section{Figure 1}

Figure 1

Figure 1. Boxplots for individual taxa that change within paired samples. The distribution of differences in paired samples is shown for phyla (A) and genera (B). Taxa with limited differences between the paired samples are tightly distributed around zero, and those with increased relative abundance after EnzD have positive distributions. Note the median RA for Fusobacteria in sputum is less than 1\%. P-values correspond to Benjamini-Hochberg corrected Wilcoxon Signed rank test for paired samples.

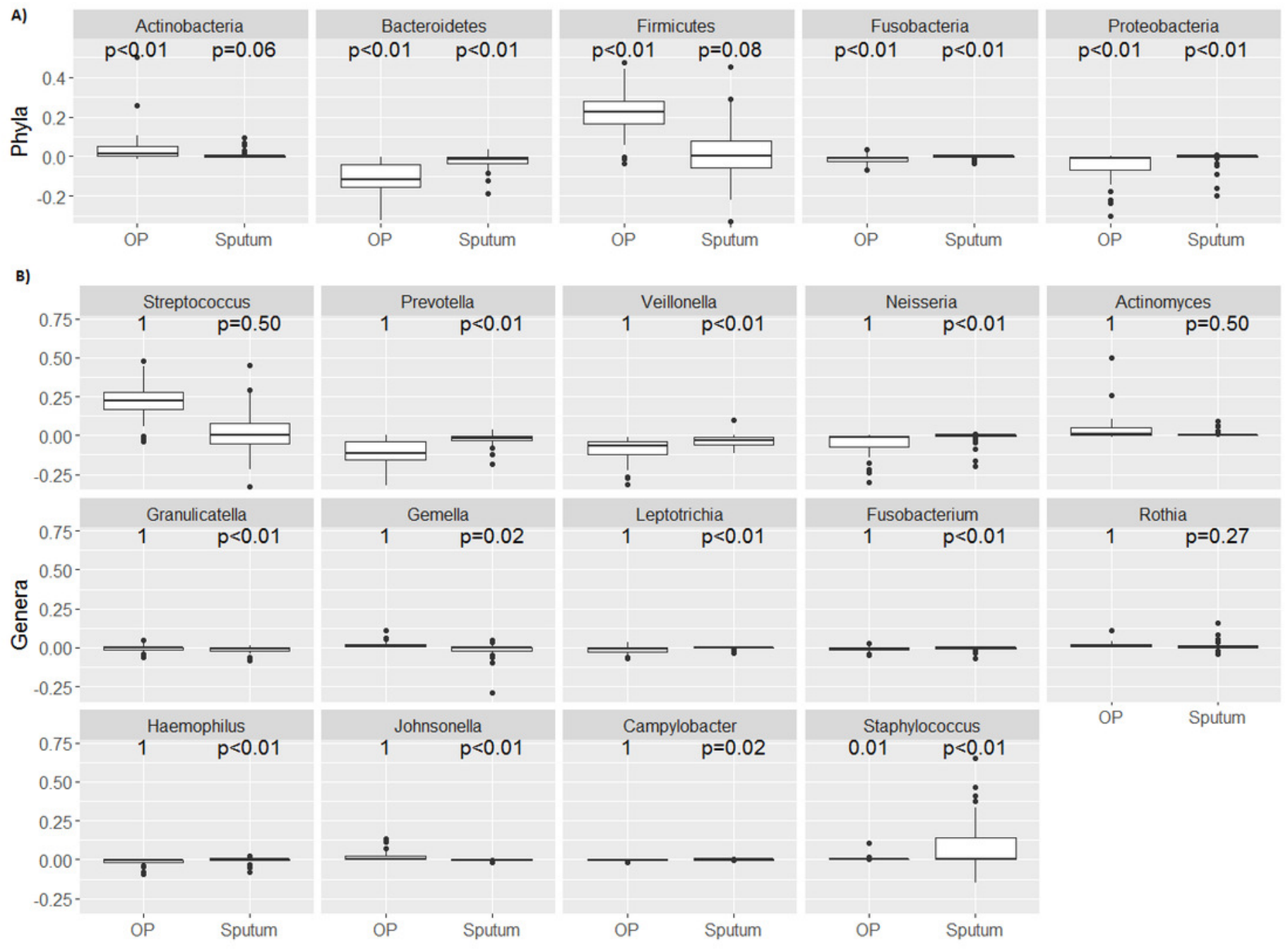




\section{Figure 2}

Figure 2

Boxplots showing distribution of Morisita-Horn (MH) similarity metric for paired samples in OP and sputum. Wilcoxon rank sum tests indicate the distributions between the two sample types were statistically different ( Benjamini-Hochberg corrected $\mathrm{p}<0.01$ ). The MH metric ranges from $0-1$ with a value of 1 indicating identical communities, and 0 indicating no overlap between communities. The line indicates the median value and the box ranges from the 25th and 75th percentiles, the whiskers extend to 1.5 times the interquartile range. 


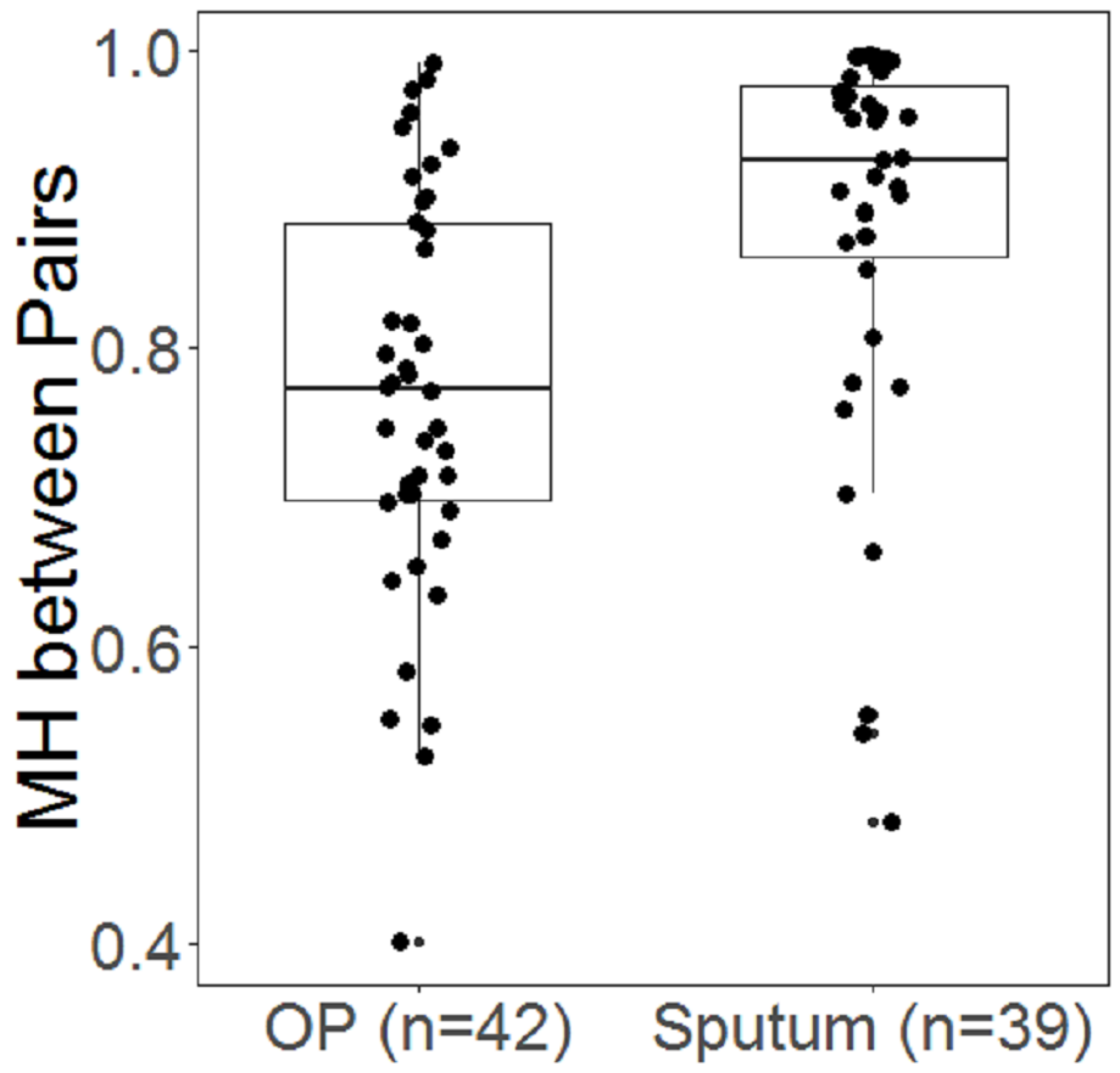


Figure 3

Figure 3

Figure 3. Ordination biplot, using 1-MH beta diversity values for $\mathrm{OP}(\mathrm{A})$ and sputum (B).

Values closer together are more similar. Paired samples are connected with a line, vectors for the genera with at least $1 \% \mathrm{RA}$ and $\mathrm{p}<0.005$ (by permutation test) for $\mathrm{R}^{\wedge} 2$ of the loading.

A)

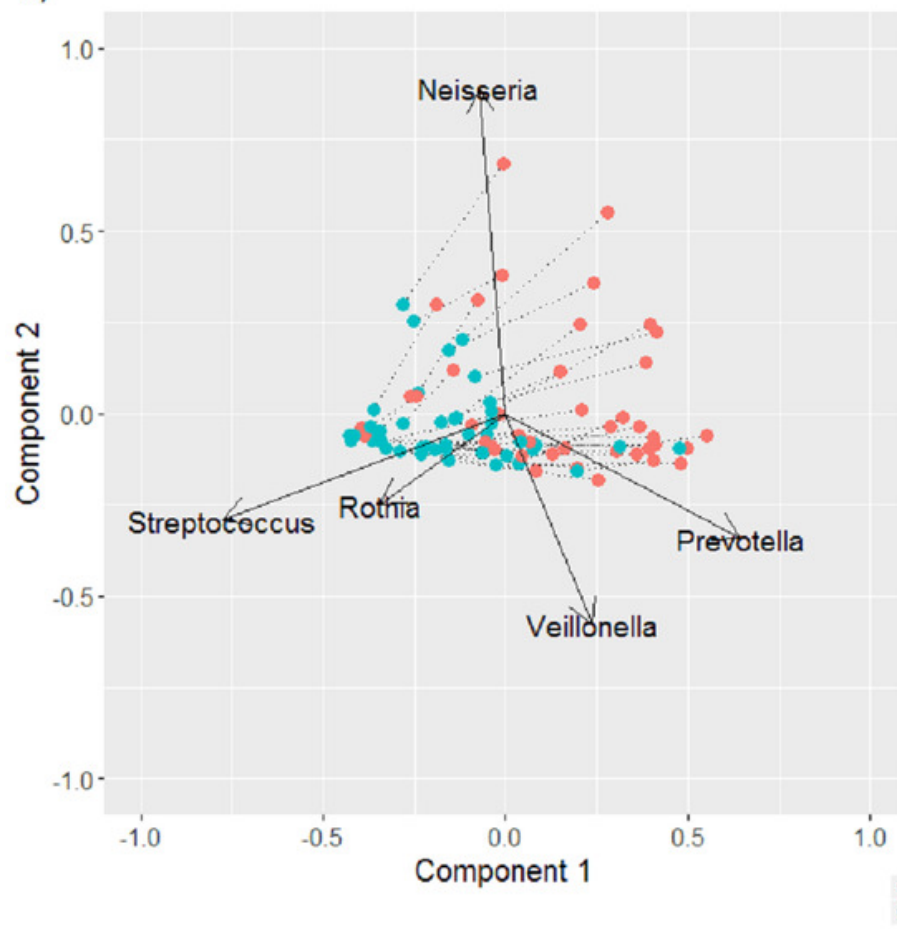

B)

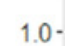

$0.5-$

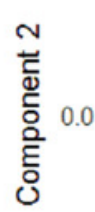

-0.5 -

(1)

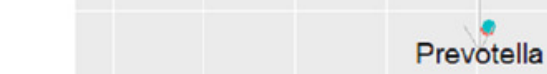

$-1.0$

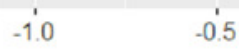

- Without EnzD - With EnzD
Streptococcus
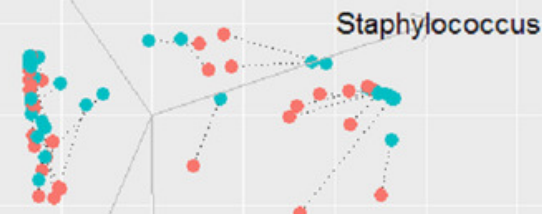

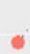

Veillonella

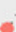

Prevotella

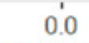

Component 1 


\section{Figure 4}

Figure 4

A) Scatterplot of relative abundance of Gram-positive taxa in non-EnzD samples versus Morisita-Horn (MH) similarity metric of paired samples. MH metric ranges from 0-1 with a value of 1 indicating identical communities, and 0 indicating no overlap between communities. OP samples are shown in red and sputum in blue. B) Scatterplot of the difference in relative abundance of Gram-positive taxa versus $\mathrm{MH}$ of paired samples OP samples are shown in red and sputum in blue.

A)

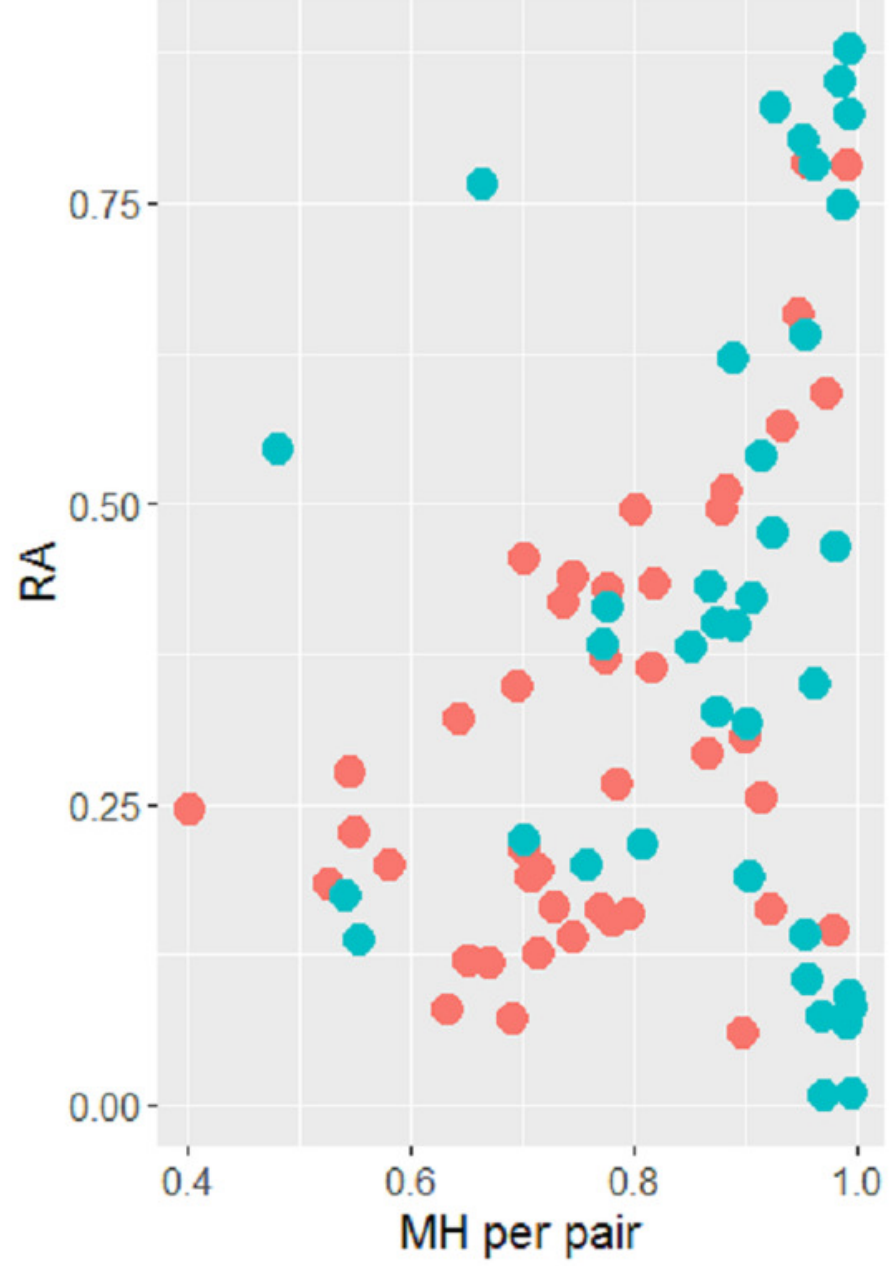

B)

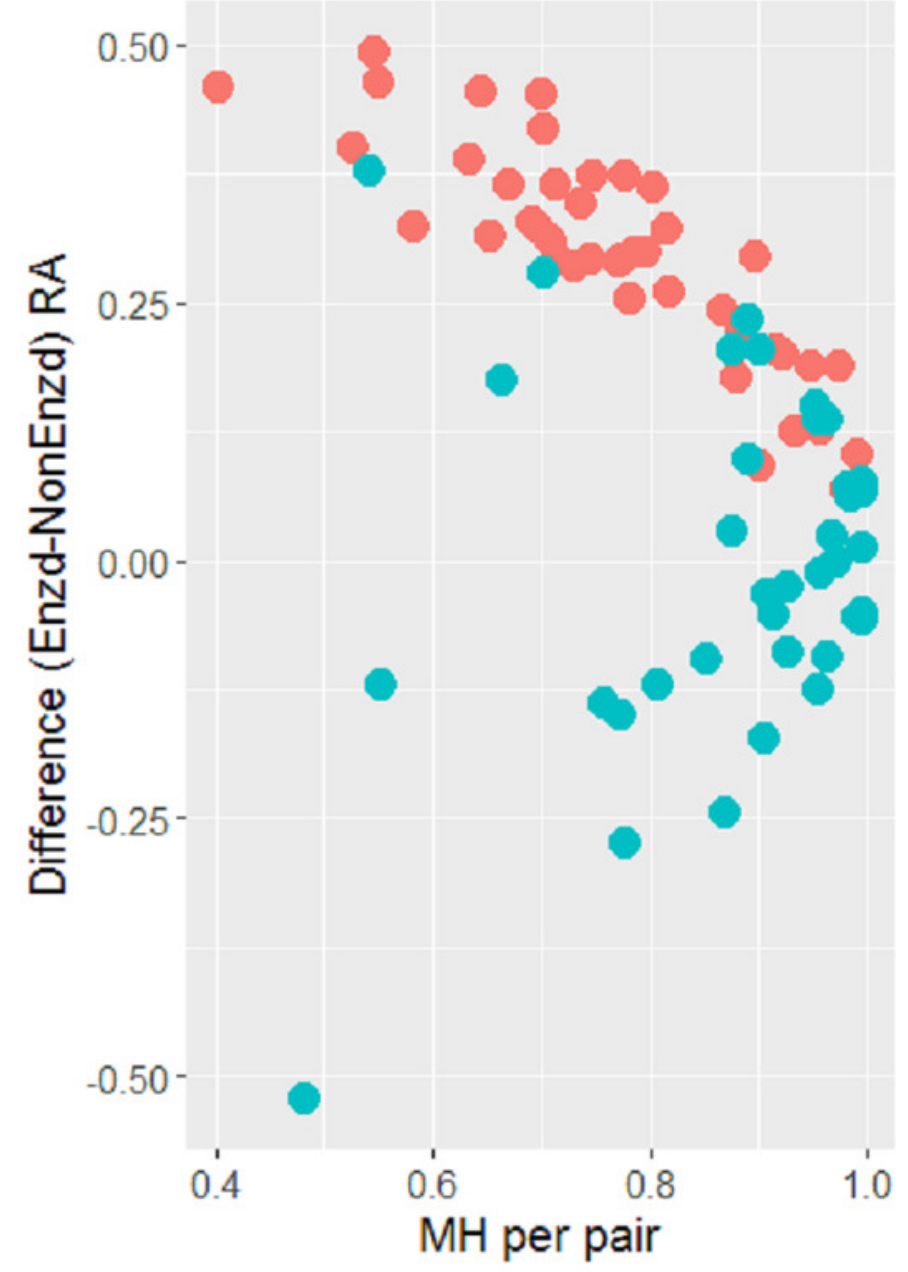

